\title{
Critical roles of long noncoding RNAs in Drosophila spermatogenesis
}

\author{
Kejia Wen, ${ }^{1,6}$ Lijuan Yang, ${ }^{1,2,6}$ Tuanlin Xiong, ${ }^{1,6}$ Chao Di, ${ }^{1}$ Danhui Ma, ${ }^{1}$ Menghua Wu, ${ }^{1}$ \\ Zhaoyu Xue, ${ }^{1}$ Xuedi Zhang, ${ }^{1}$ Li Long, ${ }^{1}$ Weimin Zhang, ${ }^{1}$ Jiaying Zhang, ${ }^{1}$ Xiaolin $\mathrm{Bi}^{3}{ }^{3}$ \\ Junbiao Dai, ${ }^{1,4}$ Qiangfeng Zhang, ${ }^{1,4,5}$ Zhi John Lu, ${ }^{1,4}$ and Guanjun Gao ${ }^{1,4}$ \\ ${ }^{1}$ MOE Key Laboratory of Bioinformatics, Tsinghua-Peking Center for Life Sciences, School of Life Sciences, Tsinghua University, Beijing \\ 100084, China; ${ }^{2}$ College of Animal Science, Tarim University, Xinjiang 843300, China; ${ }^{3}$ Institute of Cancer Stem Cell, Dalian \\ Medical University, Dalian 116044, China; ${ }^{4}$ Center for Synthetic and Systems Biology, Tsinghua University, Beijing 100084, China; \\ ${ }^{5}$ Beijing Advanced Innovation Center for Structural Biology, Tsinghua University, Beijing 100084, China
}

\begin{abstract}
Long noncoding RNAs (IncRNAs), a recently discovered class of cellular RNAs, play important roles in the regulation of many cellular developmental processes. Although IncRNAs have been systematically identified in various systems, most of them have not been functionally characterized in vivo in animal models. In this study, we identified 128 testis-specific Drosophila IncRNAs and knocked out 105 of them using an optimized three-component CRISPR/Cas9 system. Among the IncRNA knockouts, 33 (31\%) exhibited a partial or complete loss of male fertility, accompanied by visual developmental defects in late spermatogenesis. In addition, six knockouts were fully or partially rescued by transgenes in a trans configuration, indicating that those IncRNAs primarily work in trans. Furthermore, gene expression profiles for five IncRNA mutants revealed that testis-specific IncRNAs regulate global gene expression, orchestrating late male germ cell differentiation. Compared with coding genes, the testis-specific IncRNAs evolved much faster. Moreover, IncRNAs of greater functional importance exhibited higher sequence conservation, suggesting that they are under constant evolutionary selection. Collectively, our results reveal critical functions of rapidly evolving testis-specific IncRNAs in late Drosophila spermatogenesis.
\end{abstract}

[Supplemental material is available for this article.]

Thousands of long noncoding RNAs (lncRNAs; >200 nt) have been identified by genome-wide transcriptome analyses (Lee 2012; Iyer et al. 2015). In comparison to mRNA, IncRNAs are characterized by poorer conservation, lower expression levels, and more variable expression between tissues (Guttman et al. 2009; Ponting et al. 2009; Ulitsky and Bartel 2013). Deep studies have revealed the functionality of a dozen IncRNAs that play roles in biological processes such as dosage compensation, imprinting, apoptosis, immunity, cancer, and development (Pauli et al. 2011; Batista and Chang 2013; Carpenter et al. 2013). LncRNAs, such as Xist and Air, regulate transcription of neighboring genes, and thus function via a cis-acting mechanism (Lee and Bartolomei 2013; Mercer and Mattick 2013). LncRNAs have also been proposed to regulate gene expression in trans; examples include the TP53-induced lncRNAs Dlx6os1 and Hotair (Feng et al. 2006; Huarte et al. 2010; Chu et al. 2011). However, despite the huge number of IncRNAs in the genome, the biological importance of most lncRNAs remains unknown.

In multiple species, the largest repertoire of lncRNAs is expressed in the testis, as revealed by genome-wide transcriptome analyses (Djebali et al. 2012; Nam and Bartel 2012; Brown et al. 2014; Morris and Mattick 2014). Moreover, IncRNAs participate with protein-coding genes in evolutionarily conserved coexpression networks during spermatogenesis (Necsulea et al. 2014), the process by which male germline stem cells (GSCs) divide and differentiate into mature sperm in sexual organisms. To date,

\footnotetext{
${ }^{6}$ These authors contributed equally to this work. Corresponding author: gaogu@mail.tsinghua.edu.cn Article published online before print. Article, supplemental material, and publication date are at http://www.genome.org/cgi/doi/10.1101/gr.199547.115.
}

however, the functional significance of lncRNAs in spermatogenesis is unknown, with a few exceptions such as polymorphic derived intron-containing (Pldi) RNA (Heinen et al. 2009). To gain a comprehensive picture of IncRNA functionality in spermatogenesis, it is necessary to develop an efficient and large-scale gene knockout method for investigating the functions of lncRNAs in intact organisms.

The revolutionary CRISPR/Cas9-based genome editing system consists of two components-the Cas9 nuclease that cleaves DNA and the guide RNA that confers cleavage specificity (Garneau et al. 2010; Jinek et al. 2012; Wiedenheft et al. 2012). The RNA-guided DNA cleavage causes formation of double-stranded breaks (DSBs), which leads to deletions or mutations via nonhomologous end joining (NHEJ) repair (Cong et al. 2013; Mali et al. 2013). However, frameshift mutations, nonsense mutations, or small deletions caused by NHEJ are unlikely to disrupt the functions of IncRNAs. Therefore, based on a previous study by Gratz et al. (2014), we developed a three-component Cas9 microinjection system consisting of the Cas9 mRNA, a gene-specific gRNA, and a homologous recombination (HR) donor plasmid, simplifying the generation of knockout constructs; this system is applicable to deletion of almost any genomic locus. The efficiency of this system enabled us to successfully and rapidly delete 105 testis-specific Drosophila lncRNAs, 33 of which were revealed to play critical roles in the regulation of late spermatogenesis.

(c) 2016 Wen et al. This article is distributed exclusively by Cold Spring Harbor Laboratory Press for the first six months after the full-issue publication date (see http://genome.cshlp.org/site/misc/terms.xhtml). After six months, it is available under a Creative Commons License (Attribution-NonCommercial 4.0 International), as described at http://creativecommons.org/licenses/by-nc/4.0/. 
We also investigated the origin and evolution of the functionality of these 105 lncRNAs.

\section{Results \\ Systematic identification of testis-associated IncRNAs in Drosophila}

To globally characterize the biological significance of lncRNAs in spermatogenesis, we developed a stepwise selection pipeline to identify testis-specific lncRNAs in Drosophila. To this end, we first analyzed published gene expression data and identified 120 IncRNAs specifically and/or highly expressed in fly testis (Brown et al. 2014). To comprehensively identify all lncRNAs expressed in fly, we used a previously developed computational algorithm (Lu et al. 2011; Gerstein et al. 2014; Hu et al. 2015) to predict 121 novel intergenic IncRNAs with no overlap with protein-coding genes; the predictions were based on RNA-seq data and RNA structure information (Fig. 1A; Supplemental Fig. S1A). From the resultant 241 lncRNAs, we ultimately identified the 128 testisspecific IncRNAs from a testis-specific expression screen and RNA in situ hybridization analysis. These lncRNAs were located on three different chromosomes (Fig. 1A). Meanwhile, our RTPCR and lncRNA in situ hybridization results indicated that they were highly or specifically expressed in the Drosophila testis (Fig. 1B,C; Supplemental Fig. S1B,C). The majority of testis-specific IncRNAs were strictly expressed in the meiotic and post-meiotic region of the testis (Fig. 1C; Supplemental Fig. S2). Thus, we identified 128 testis-specific lncRNAs for further targeted mutational analysis.

\section{The development of a three-component CRISPR/Cas9 system streamlines IncRNA gene knockout in Drosophila}

To comprehensively analyze lncRNA functions in vivo, we need an efficient and robust experimental system to inactivate IncRNA genes by genomic deletion. Considering that the point mutations, small deletions, or inversions induced by a single gRNA are unlikely to disrupt lncRNA function unless the gRNA is targeted based on prior knowledge of the functional domains (Sauvageau et al. 2013; Yin et al. 2015), we tried to knock out an entire IncRNA gene by replacing it with an RFP marker. To this end, we developed an efficient three-component CRISPR system, including Cas 9 mRNA, gRNA, and an HR donor plasmid, to perform precisely targeted deletions via homologous recombination (Fig. 2A; Supplemental Fig. S3A).

To optimize the system for scalable genome-wide deletion analysis, we investigated three factors that might potentially affect HR efficiency, including the length of donor homology arms, the absence of NHEJ, and the distance between DSBs and homology arms. We first used the system to knock out the wellstudied protein-coding gene oskar (Lehmann and NüssleinVolhard 1986). Donor plasmids containing homology arms with lengths of 1.5 or $3 \mathrm{~kb}$, but not $0.3 \mathrm{~kb}$, were sufficient to generate a 5-kb deletion in oskar (Fig. 2B; Supplemental Fig. S3B,C). Deletion in oskar caused defects in oocyte development and complete sterility in females, as previously reported (Lehmann and Nüsslein-Volhard 1986). To further test this strategy for large fragment deletions using 1.5-kb homology arms, we tried to knockout a $92-\mathrm{kb}$ fragment in a known lncRNA gene, iab-8, in the genome. Surprisingly, one correct HR targeting event was obtained from
40 fertile crosses (Supplemental Fig. S3D). The iab-8 knockout was pupa lethal.

In mammalian cells, inhibition of the NHEJ pathway can increase HR efficiency (Maruyama et al. 2015; Yu et al. 2015). To determine whether this is also the case in Drosophila, we compared the knockout efficiencies of five IncRNA genes in wild-type and $\mathrm{Lig}^{-/-}$mutant flies. The Lig4 deletion did not dramatically increase HR frequencies in any of the tested knockouts, suggesting that inhibition of NHEJ has a negligible effect on HR efficiency in the fly (Fig. 2C). Furthermore, the distance between the doublestrand break site and the homology arms had no dramatic effect on HR frequency (Fig. 2D). Knockout analyses of 18 lncRNAs indicated that HR frequencies were similar for HR donor arms adjacent to the breaks and for arms $700 \mathrm{bp}$ away from the break sites.

Last, we developed a new high-throughput cloning strategy for constructing plasmids carrying homology arms in 96-well plates (Supplemental Fig. S3E). Using this system, we successfully generated deletion strains corresponding to 105 of the 128 testisspecific lncRNAs identified as described above (Supplemental Table S1). The deletions were confirmed independently by PCR and sequencing (Fig. 2E; Supplemental Fig. S4). The deleted genomic regions ranged from 200 to $11 \mathrm{~kb}$ (Fig. 2F; Supplemental Table S1), and the HR efficiency was as high as 47\% (Fig. 2G; Supplemental Table S1). As expected, all 105 homozygous IncRNA deletion strains were viable, because these RNAs are primarily expressed in late germ cells of the Drosophila testis.

To determine whether our CRISPR/Cas9 knockout system induced off-target mutagenesis by introducing DSBs at unintended genomic sequences, we performed PCR amplification and sequencing analysis of all potential off-target cleavage sites in 22 lncRNA knockout mutants (see online tool, http://crispr.mit. edu). We did not detect mutations at any of these sites (Supplemental Fig. S5). Taken together, these results indicate that our three-component CRISPR-based knockout system is suitable for rapid large-scale functional investigation of lncRNA genes in vivo.

\section{Some testis-specific IncRNAs are required for male fertility and late spermatogenesis}

Given the testis-specific expression of the lncRNAs we identified, we examined the fertility of knockout (KO) mutant males using a standard fertility test (Sitnik et al. 2014). After virgin females mated with wild-type control or lncRNA knockout males, the numbers of progeny from each female fly were counted daily over a 15-d period. In 32 of 105 lncRNA knockouts (30\%), we observed a substantial reduction in male fertility (Fig. 3A; Supplemental Fig. S6). One IncRNA KO $\left(C R 44455 / 6^{-/-}\right)$strain had a more severe phenotype and was completely male-sterile (Fig. 3B,C). In contrast, lncRNA $\mathrm{KO}$ mutant females did not exhibit any obvious reduction in fertility (Fig. 3B,C). These results suggested that $30 \%$ of the testis-specific IncRNAs we identified have important functions during Drosophila spermatogenesis.

To further characterize KO phenotypes, we microscopically examined testis morphology and mature sperm in seminal vesicles of lncRNA KO males. Ten of the 33 deletion mutants with diminished male fertility, including CR44455/6 ${ }^{-1-}, \operatorname{lncRNA} T$ TS $23^{-1-}$, and $C R 43282^{-/-}$, exhibited severe morphological defects in testis. The abnormalities included an accumulation of cotton-like white flocculus in the distal part of the testis, which corresponds to the later stages of sperm development (Fig. 4A; Supplemental Fig. S7A). The flocculus contained a large number of tightly packed small cells (Supplemental Fig. S7B). In addition, the sperm in seminal vesicles

\section{Genome Research}

www.genome.org 
A
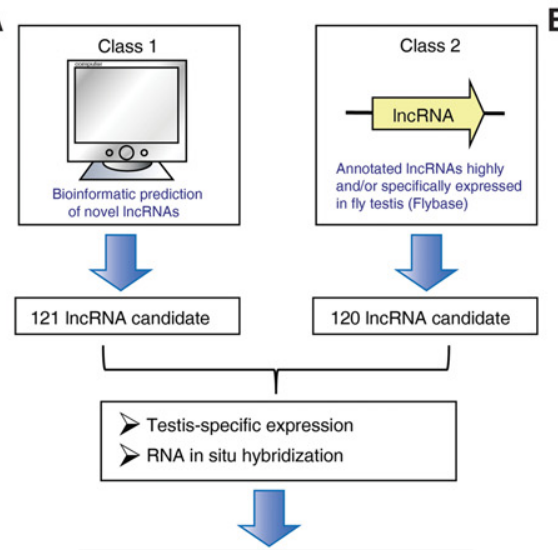

128 IncRNA candidates for knock-out screen

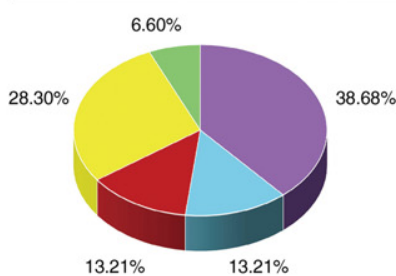

chromosome 2L chromosome 2R

- chromosome 3L chromosome 3R chromosome X

C

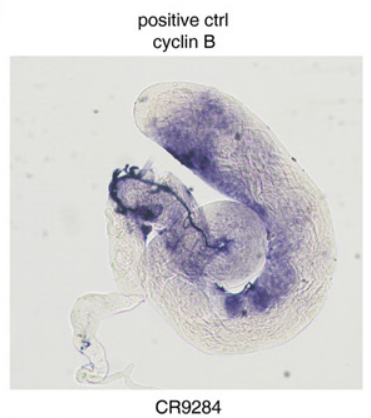

CR9284

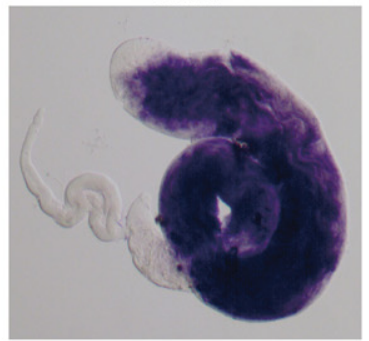

B

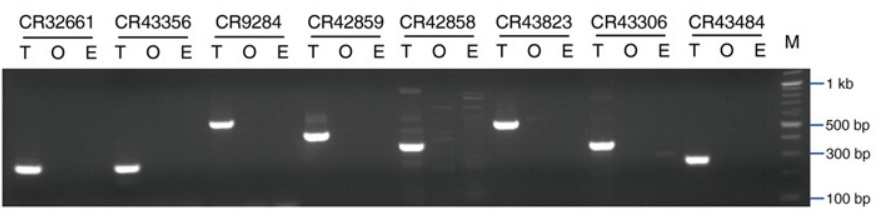

Positive ctrl Negative ctrl Testis ctrl

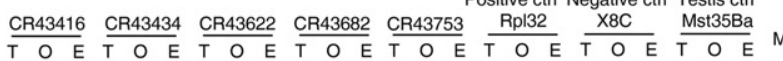

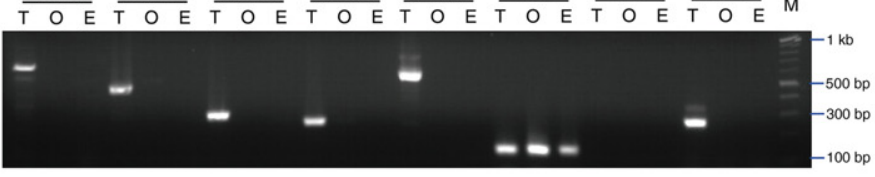

T-Testis, O-Ovary, E-0-2 h Embryo

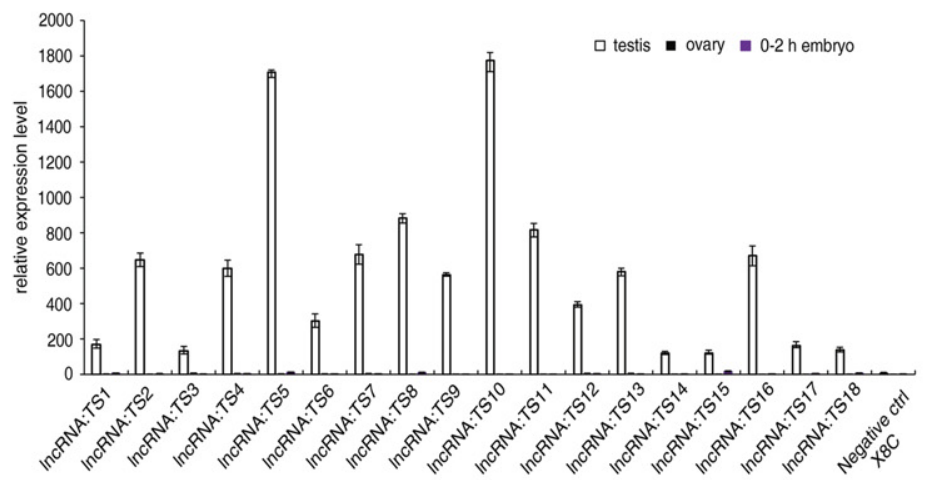

CR42858

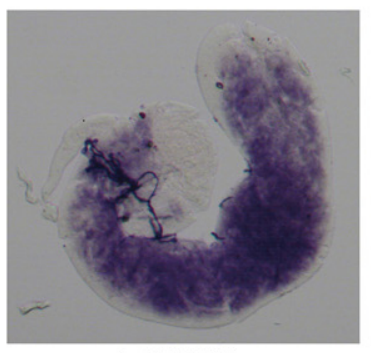

IncRNA:TS21

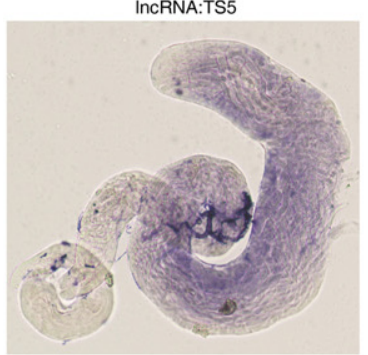

RFP
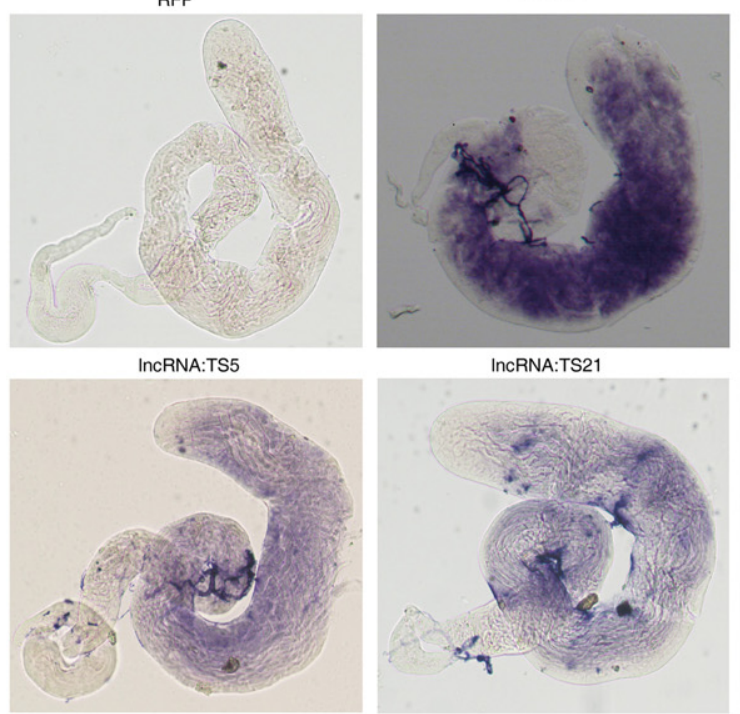

CR32661

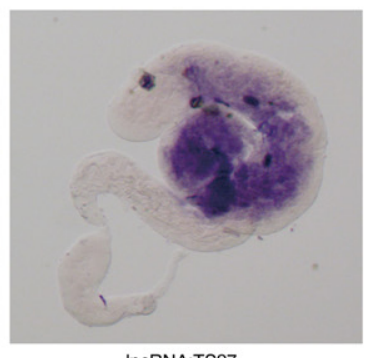

IncRNA:TS27

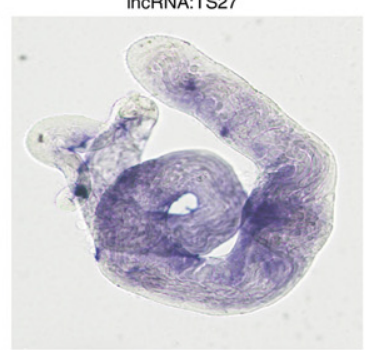

Figure 1. Systematic identification and validation of Drosophila IncRNAs involved in spermatogenesis. (A) Flowchart of identification and selection of testis-specific IncRNAs for the knockout study. Novel IncRNA prediction using bioinformatics and analysis of annotated IncRNAs in FlyBase were combined to build the IncRNA starting pool. Then, through a testis-specific expression screen and RNA in situ hybridization, 128 testis-specific IncRNA candidates were selected for targeted knockout. These IncRNAs were located on three different chromosomes, including the left and right arms of Chromosome 2, the left and right arms of Chromosome 3, and Chromosome X. (B) Testis-specific expression screen of predicted IncRNAs and annotated IncRNAs by quantitative RT-PCR and semiquantitative RT-PCR, respectively. RpL32 was used as an internal control. Values represent means \pm SEM for three biological replicates. Mst35Ba was used as a testis-specific control. X8C (a Chromosome X-linked intergenic region that has been determined to be silent for transcription) was used as a negative control to rule out contamination of RNA by genomic DNA. (C) Expression of selected IncRNAs in Drosophila testis, analyzed by whole-mount in situ hybridization. Cyclin B RNA was used as a positive control, and RFP RNA in nontransgenic testis (w1118) was used as a negative control.

of $C R 44455 / 6^{-1-}$ males were smaller than those in wild-type flies (Fig. 4A). Other lncRNA mutants with severe white flocculus, such as CR42858 ${ }^{-/-}$and IncRNA:TS23 ${ }^{-/-}$, contained significantly smaller numbers of mobile mature spermatozoa in their seminal vesicles (Fig. 4A; Supplemental Fig. S7A). These observations explain the reduced male fertility of some lncRNA deletion mutants. 
A

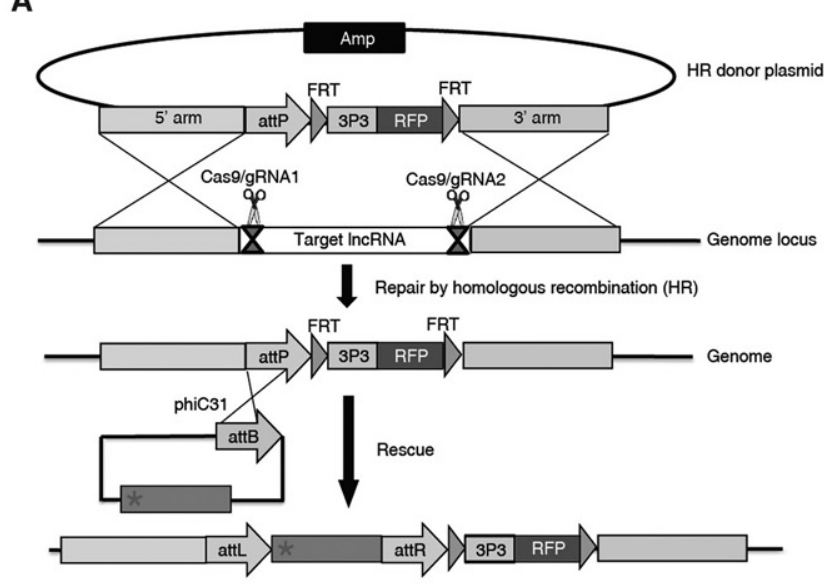

C

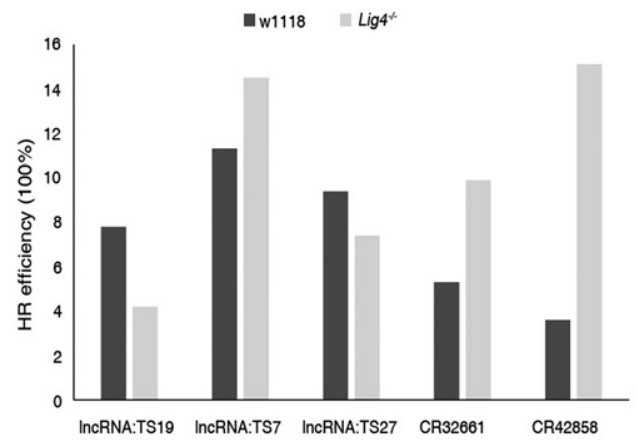

E

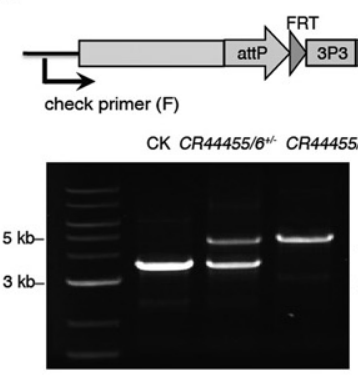

RFP
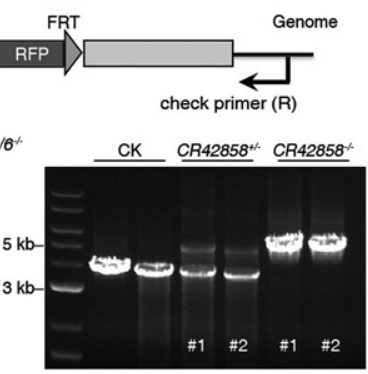

B

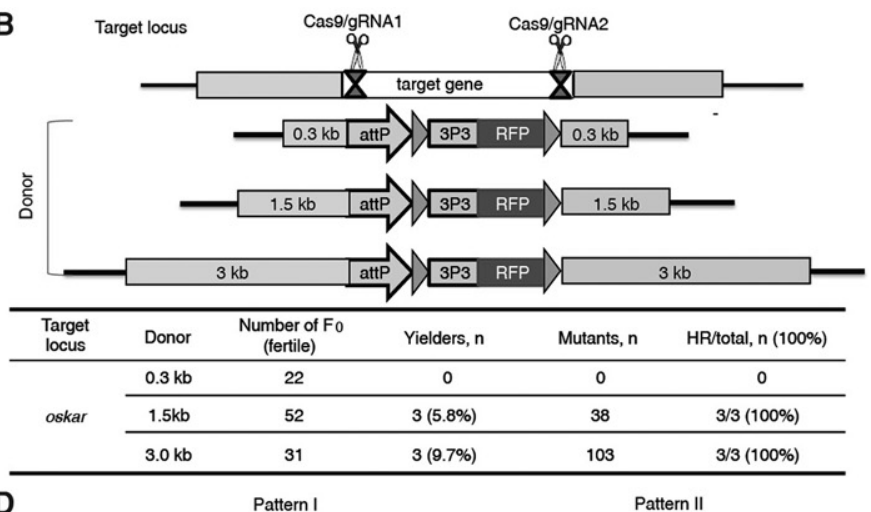

D
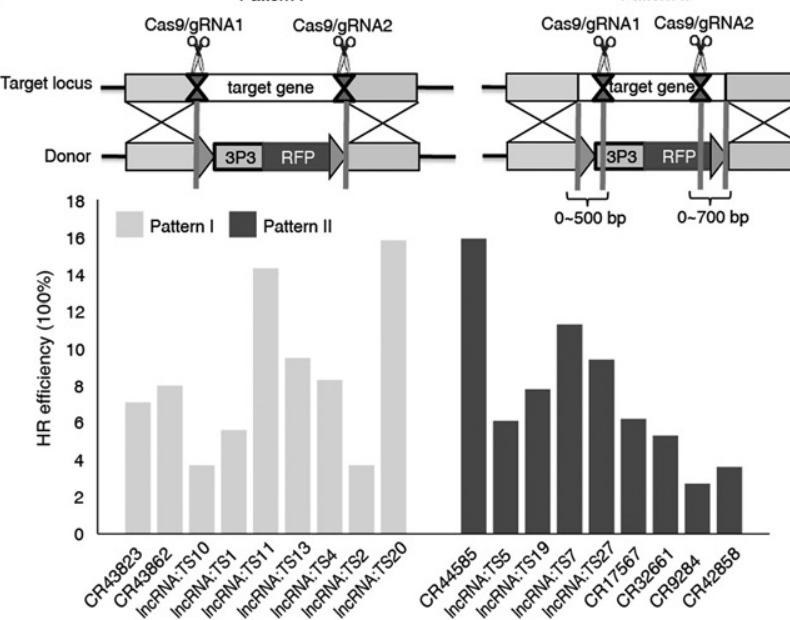

F

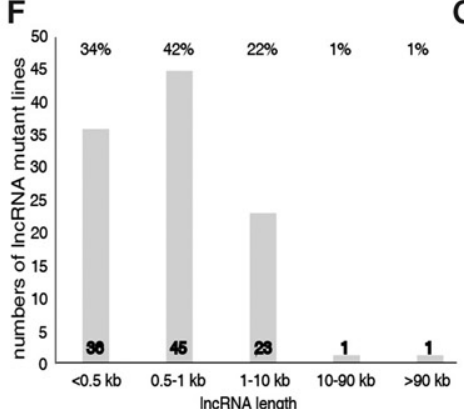

G

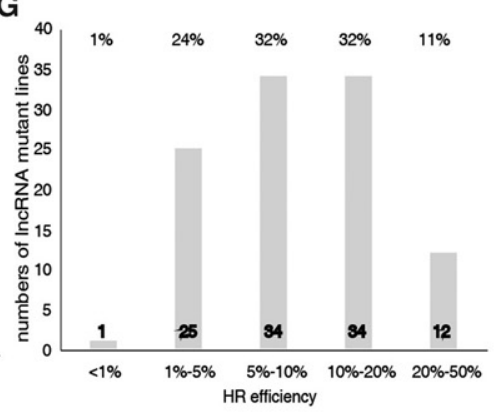

Figure 2. CRISPR/Cas9-mediated high-throughput mutagenesis of 105 IncRNAs. (A) Schematic representation of the strategy for generating IncRNA knockout and rescue lines using CRISPR. (B) Determination of the optimal HR donor length for efficient gene replacement. The plasmid containing a reporter cassette (attP-FRT-3P3-RFP) flanked by $0.3,1.5$, and $3 \mathrm{~kb}$ HR sequences was provided as the donor DNA. The oskargene was used to test the system. (C) DNA Ligase4 deficiency had no dramatic effect on HR efficiency at different target loci. $(D)$ Within a range of 0-700 bp, the distance between the double-strand break site and the homology arm had no dramatic effect on HR frequency. (E) Genotyping of w1118, IncRNA $A^{+-}$, and IncRNA ${ }^{-/-}$flies to confirm IncRNA knockouts. Insertion of the reporter cassette results in a size increase in the PCR products, which is visualized only in heterozygous and homozygous flies. $(F)$ Length distribution of the targeted IncRNAs. $(G)$ The distribution of HR efficiency when generating the 105 IncRNA mutants using CRISPR. $\mathrm{F}_{0}$ injected flies were crossed to w1118, and their progeny were screened for RFP-positive eyes. Crosses producing one or more transgenic progeny were considered as founder lines. The homologous recombination efficiency was calculated as the ratio between the number of founder lines and the number of $F_{0} c r o s s e s$.

Testis-specific IncRNAs are required for nuclear condensation and morphogenesis

To further characterize any possible defect in late germ cell development in lncRNA KO testes, we examined spermatid morphology of the 105 lncRNA KO mutants by testis squash and DAPI staining. During spermatid development, the initially round nuclei synchronously elongate and condense to form long, straight, needle-shaped structures. Twenty-two of the IncRNA KO mutants lost synchronization and exhibited defects in spermatid morphogenesis (Fig. 4B,C; Supplemental Fig. S8). The developmental defects could be classified into three major types. First, $\sim 10 \%$ of spermatids in IncRNA:TS1 ${ }^{-/-}$and $\sim 20 \%$ of sperm heads in CR43484 ${ }^{-/-}$testes adopted a tadpole shape, in which the sperm nucleus was concentrated at one end of the cell (Fig. 4B; Supplemental Fig. S8). This phenotype is similar to those of protamine mutations (Rathke et al. 2010). Second, CR45542 $2^{-1-}$ and CR44420 ${ }^{-/-}$mutant testes contained some round uncondensed

\section{Genome Research}

www.genome.org 

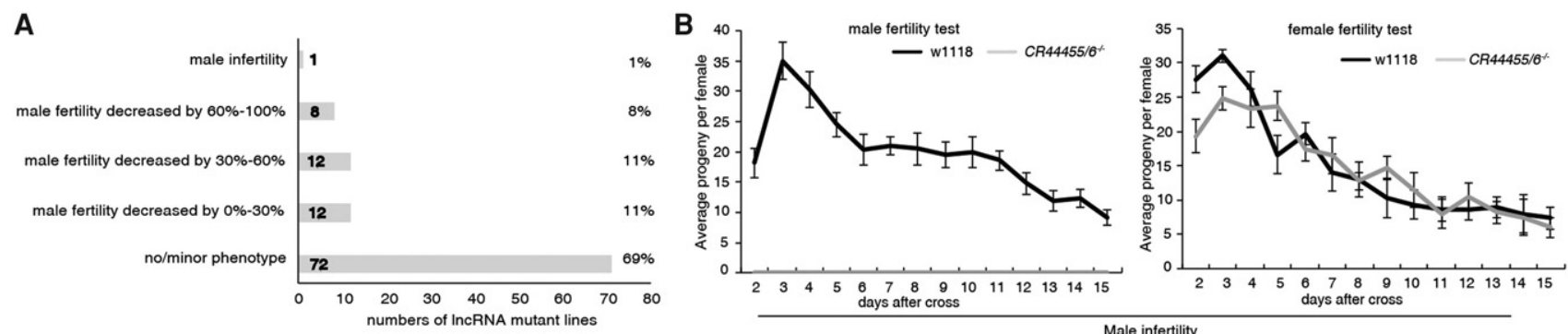

C
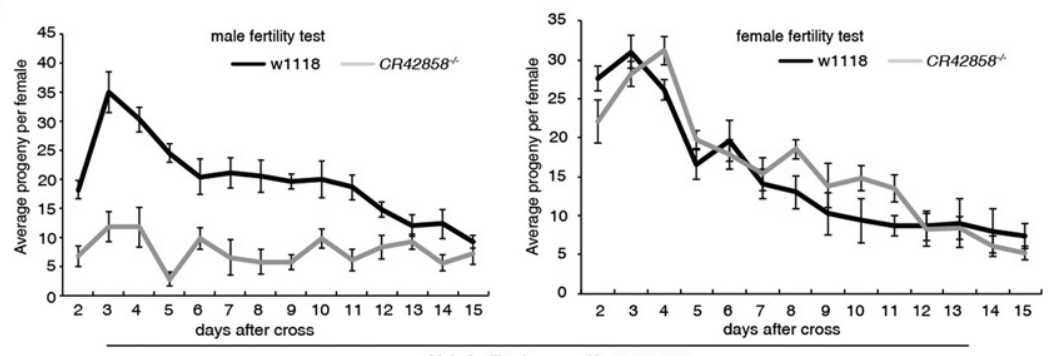

Male fertility decreased by $30 \%-60 \%$

Figure 3. IncRNA mutants develop male-specific fertility defects. (A) Fertility profiles of 105 IncRNA mutants. (B) Deletion of IncRNA CR44455/6 results in male sterility, whereas $C R 44455 / 6^{-1-}$ females were fully fertile. (C) A qualitative fertility assay was performed for both male and female mutant flies of CR42858 $8^{-/-}$. Deletion of CR42858 substantially reduced fertility in males, but not in females. Values represent means \pm SEM for 15 crosses each.

nuclei ( $30 \%$ in CR45542 ${ }^{-/-}$and $\sim 20 \%$ in $C R 44420^{-/-}$) at the mature stage (Fig. 4B,C), indicating that these two lncRNAs are required for nuclear condensation. Third, 17 other IncRNA mutants, including CR42858 ${ }^{-1-}$ and $C R 43416^{-/-}$, had scattered or curled sperm ( 25\% in CR42858 ${ }^{-/-}$and $\sim 30 \%$ in $C R 43416^{-1-}$ ) (Fig. 4B,C). This phenotype is reminiscent of mutants in the testis-specific proteasome subunit Prosa6T (Zhong and Belote 2007) or yuri (Texada et al. 2008).

Next, we used HIS2AV-RFP and Protamine B-GFP to mark early spermatids and mature sperm, respectively (Rathke et al. 2007, 2010). The round uncondensed sperm of $C R 44420^{-/-}$, tadpoleshape sperm of $\operatorname{lncRNA} T S 1^{-/-}$, and bent sperm of CR43416 ${ }^{-/-}$ were positive for Protamine B-GFP but not HIS2AV-RFP, confirming that they corresponded to the late elongate and/or mature stage (Fig. 4C). This result is consistent with a previous study of Prosa6T (Zhong and Belote 2007). Surprisingly, the mature sperm in the seminal vesicles of these lncRNA mutants did not have abnormal morphologies, suggesting that mutant sperm with abnormally condensed chromatin might not enter the seminal vesicle.

In contrast to the spermatid nuclear phenotype described above, the spermatids in $C R 44455 / 6^{-/-}$mutant testis were smaller than those of the wild type from the meiotic stage onward (Fig. 4D); specifically, mature $C R 44455 / 6^{-1-}$ sperm were about half the size of wild-type sperm. The small nuclei in the mutant could be caused by overcondensation. Because CR44455/6 ${ }^{-1-}$ mutant males were completely sterile, these small mature sperm were defective in fertilization. Taken together, these data indicate that some of the testis-specific lncRNAs play important roles in controlling proper nuclear condensation during late spermatogenesis.

\section{Some IncRNAs regulate spermatid individualization}

In spermatogenesis, after completion of meiosis, 64 cells in each spermatid cyst begin differentiating into individual sperm. Once fully elongated, spermatids undergo the process of individualization, which separates individual sperm tails and removes excess cy- toplasm (Ma et al. 2010). During individualization, the actin-based investment cones (ICs) form and translocate mature spermatid nuclei down axonemes. To obtain insight into the nature of the spermatogenesis defects of lncRNA mutants, we further analyzed ICs by labeling testes for actin bundles, myosin VI, and DNA. In wild-type testes, ICs assemble above spermatid nuclei and coordinately move along spermatid bundles as a complex. However, the testes of 19 lncRNA mutants contained poorly aligned or lagging ICs (Fig. 4E; Supplemental Fig. S9). In the testes of the CR43282 ${ }^{-/-}, C R 42859^{-/-}$, and CR44371 $1^{-/-}$mutants, the ICs were severely disorganized, and the actin cone structures were scattered (Fig. 4E; Supplemental Fig. S9). In the CR43484 ${ }^{-/}$and CR44420 ${ }^{-/-}$mutants, the nuclei failed to remain tightly clustered and were displaced distally along the cyst, resulting in lagging ICs (Fig. 4E; Supplemental Fig. S9). These observations indicate that some of the testis-specific lncRNAs are required for developmental synchronization of the 64-cell cyst and the shaping and differentiating of spermatids during late spermatogenesis.

\section{Testis-specific IncRNAs function in trans to regulate late spermatogenesis}

To rule out the possibility that the phenotypes of lncRNA mutants were caused by off-target events, we first performed in cis rescue experiments on CR42858, CR43484, CR44585, IncRNA:TS2, CR43416, and CR43862 deletion mutants by inserting the wildtype lncRNAs under the control of their endogenous promoters via PhiC31-mediated attB/attP exchange (Fig. 5A; Supplemental Fig. S10). The late spermatogenesis defects of these six lncRNA deletion mutants were rescued by in cis lncRNA restoration, indicating that the late spermatogenesis phenotypes were indeed caused by deletion of the IncRNAs (Fig. 5A). To determine whether these lncRNAs function in trans, we transgenically rescued the CR42858, CR43484, CR44585, IncRNA:TS2, CR43416, and CR43862 deletion mutants by expressing the corresponding 
A

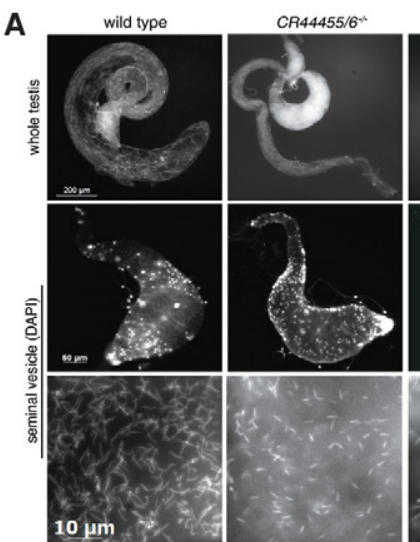

C
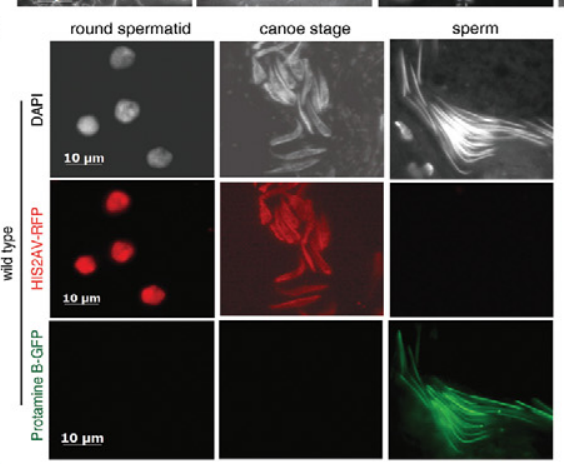

D

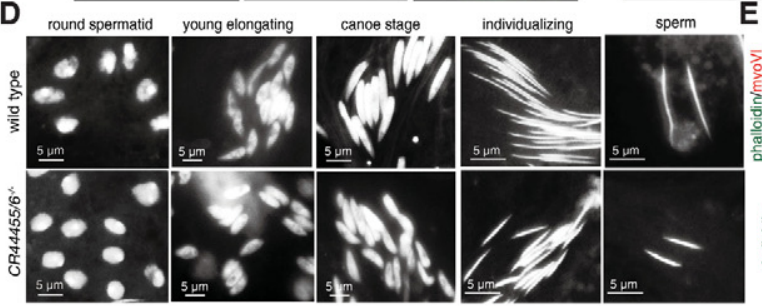

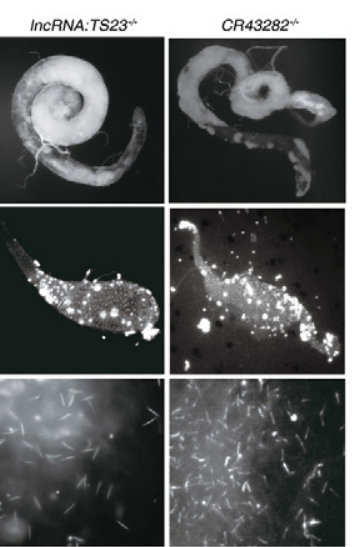

round spermatid
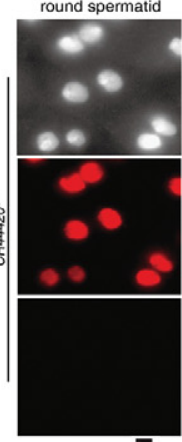

E
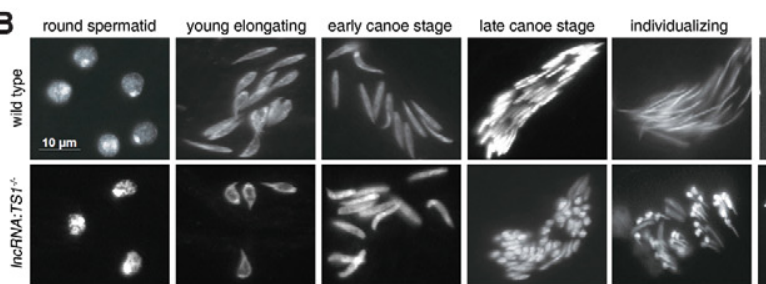
sperm

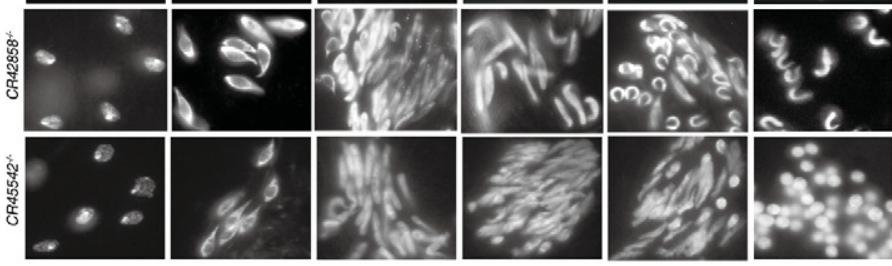

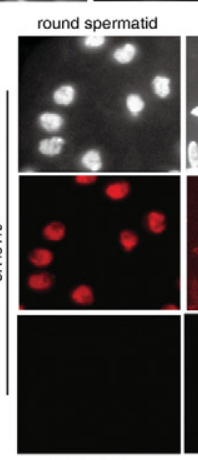

canoe stage sperm

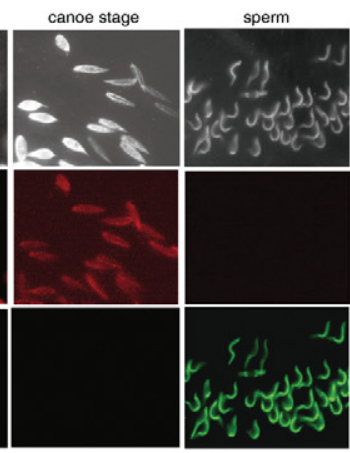

CR43484, canoe stage
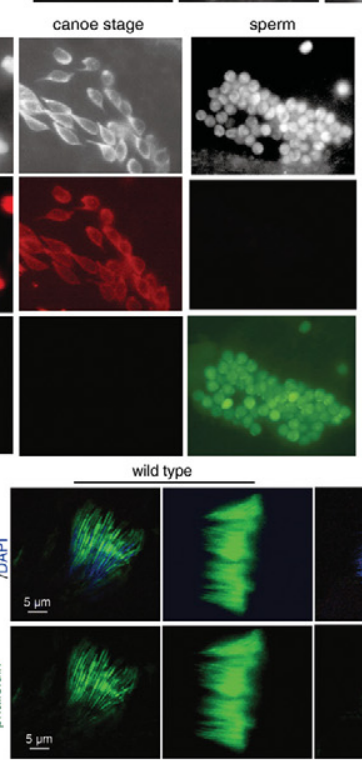

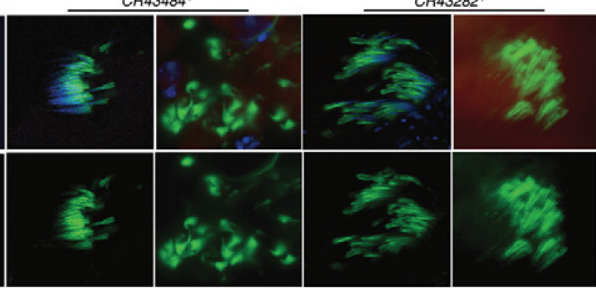

Figure 4. Deletion of IncRNAs lead to various defects in spermatogenesis. $(A)$ LncRNA knockout mutants cause malformation and obstruction of the testis: (top rows) whole testis; (middle rows) seminal vesicle stained with DAPI; (bottom rows) sperm in seminal vesicle. Scale bars are noted. Testes of CR44455 $/ 6^{-1-}$, InCRNA:TS23 ${ }^{-1-}$, and CR43282 $2^{-1-}$ contained an accumulation of abnormal white flocculus, although these IncRNA mutants had a normal spherical testis shape. Seminal vesicles from $C R 44455 / 6^{-1-}$ males contained smaller sperm relative to wild type. IncRNA:TS23 ${ }^{-/-}$contained significantly reduced numbers of mature sperm in seminal vesicles, whereas the numbers in $C R 43282^{-/-}$were comparable to those in the wild type. ( $B$ ) LncRNA knockouts affect male germ cell development. Testes squash preparations were stained with DAPI to visualize DNA of the wild-type and IncRNA mutants. In the wild type, the initially round spermatids nuclei elongated and condensed to form long, straight, and needle-shaped mature sperm. In InCRNA:TS1 ${ }^{-1-}$, some mature-stage sperm adopted a tadpole shape in which the nucleus was concentrated at one end of the spermatid head. Deletion of CR42858 led to scattered or curled sperm in which some nuclei did not fully condense. CR45542 ${ }^{-/-}$exhibited some round uncondensed sperm at a very mature stage. (C) Chromatin condensation defects of IncRNA mutants appear in late spermatogenesis. HIS2AV-RFP and Protamine BGFP were used to distinguish early spermatid nuclei and mature sperm, respectively. The round uncondensed nuclei of $C R 44420^{-1-}$ and the bent sperm of $C R 43416^{-l-}$ were labeled by Protamine B-GFP but not HIS2AV-RFP, indicating that these abnormal germline cell phenotypes appeared in late elongate to mature sperm stage. (D) Spermatogenesis in wild type and $C R 44455 / 6^{-1-}$ (male infertility). Spermatids in $C R 44455 / 6^{-1-}$ were smaller than those in the wild type from the meiotic stages onward. The nuclei of mature sperm in CR44455/6 ${ }^{-1-}$ were half the size of those in wild-type sperm. (E) LncRNA mutants exhibit individualization defects. Phalloidin was used to stain investment cones (ICs) in wild-type and IncRNA mutants. Wild-type testis contained ordered and associated ICs. In CR43484 $4^{-1-}$ and CR43282 ${ }^{-1-}$, ICs were severely disorganized or lagged, and individual actin cone structures were scattered. Scale bars are noted.

lncRNAs on a different chromosome via PhiC31-mediated attB/ attP exchange. The spermatogenesis defects of all six lncRNA mutants were also rescued by expression of the lncRNAs in trans, ruling out the possibility that the phenotypes of the deletions are caused by the disruption of the regulatory DNA elements (Fig. $5 \mathrm{~A})$. These results indicate that these testis-specific lncRNAs primarily function in trans to regulate late spermatogenesis.

Next, we investigated the function of the IncRNA CR42858 in more detail. CR42858 was efficiently transcribed from transgenes either at its endogenous locus (in cis) or a different chromosome (in trans) (Fig. 5B). The abnormal testis morphology and poorly aligned IC phenotypes of $C R 42858^{-/-}$were rescued by transgenes in cis and in trans (Fig. 5C). To further separate RNA from DNA sequence-dependent effects, we individually introduced CR42858 DNA sequences without the promoter and CR42858 promoterdriven eGFP sequences into CR42858 mutant flies in situ (Supplemental Fig. S11). The phenotypic defects in $C R 42858^{-/-}$ were not rescued by CR42858 DNA without the promoter or by CR42858 promoter-driven eGFP (Supplemental Fig. S11). All these results rule out the possibility that the defective phenotypes of

\section{Genome Research}

www.genome.org 
A
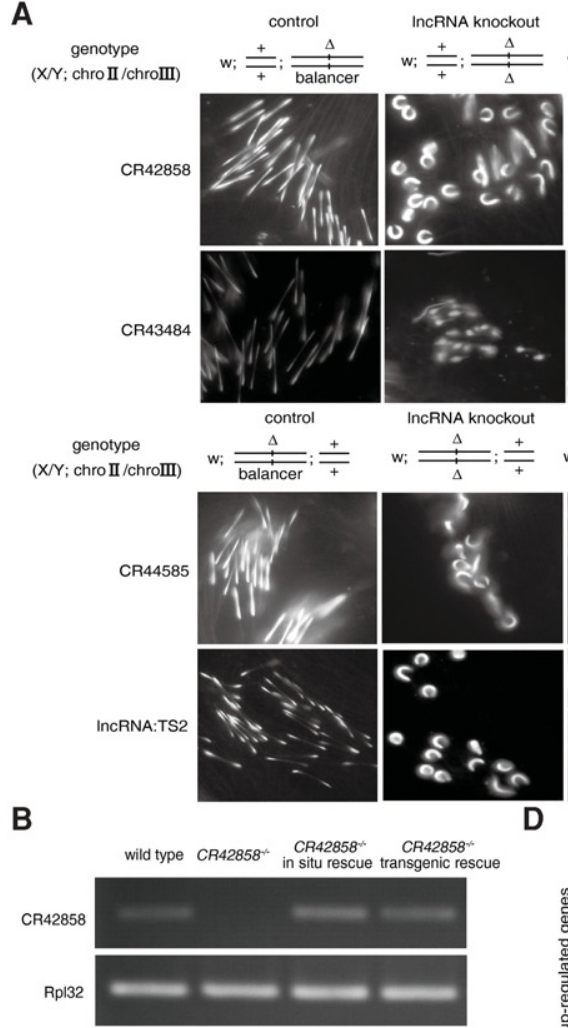

E

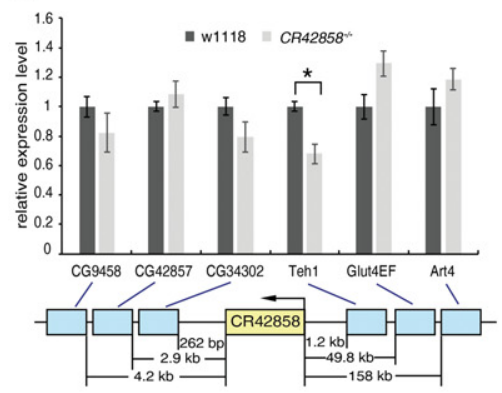

IncRNA in situ rescue IncRNA transgenic rescue C
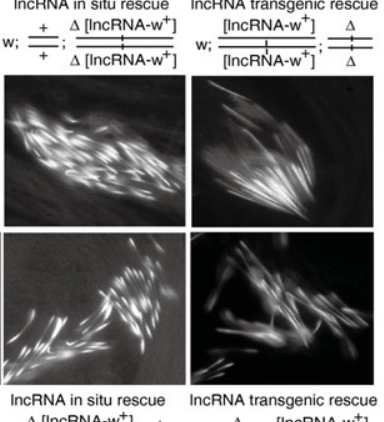

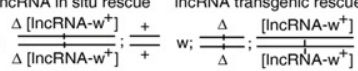
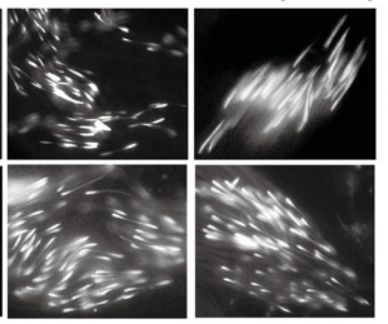

(1)

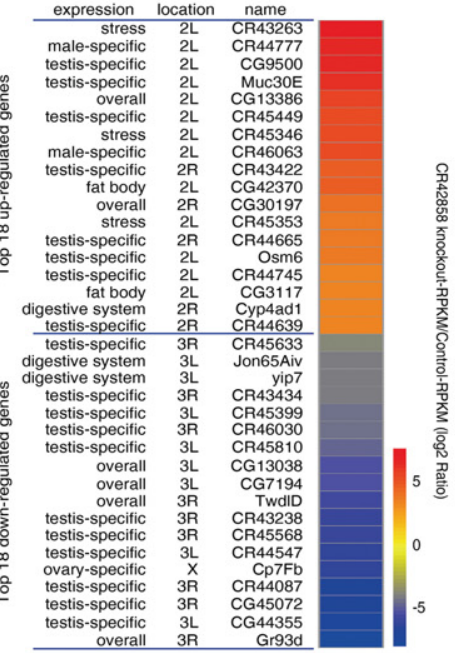

C wild type
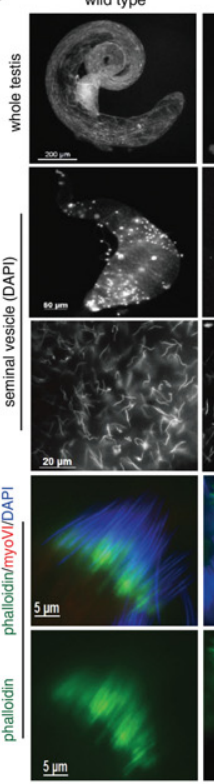

$\mathbf{F}$
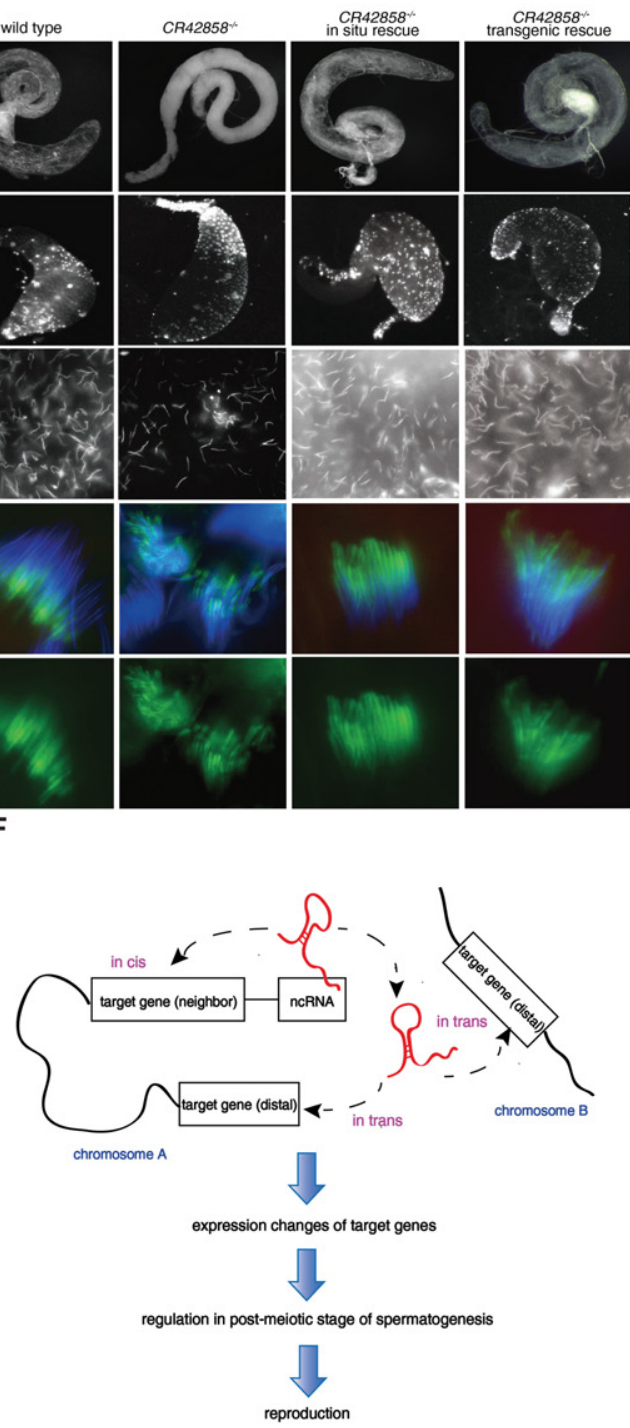

Figure 5. Testis-specific IncRNAs function in trans to regulate late spermatogenesis. (A) Male germ cell development defects of four IncRNA knockout mutants were rescued by restoration of the corresponding IncRNAs in cis or in trans. For in cis rescue, a construct bearing an IncRNA gene fragment under the control of the endogenous promoter was inserted back into the genomic locus from which the original IncRNA had been deleted. For rescue in trans, the IncRNA was placed on another chromosome (i.e., different from the original chromosomal locus of the IncRNA) through PhiC31-mediated attB/attP exchange. The abnormal morphological phenotype of scattered and curled sperm nuclei in late spermatogenesis of $C R 42858^{-1-}, C R 43484^{-I-}$, CR44585 $5^{-1-}$, and InCRNA:TS2 $2^{-1}$ were rescued by restoration of these IncRNAs either in cis or in trans. (B) LncRNA CR42858 was transcribed in flies rescued with CR42858 in cis and in trans, but not in CR42858 ${ }^{-1-}$. RNA was isolated from the testes of wild-type, CR42858 ${ }^{-1-}$, and flies rescued with CR42858 in cis or in trans, and subjected to semiquantitative RT-PCR. RpL32 was used as a control. (C) In cis or in trans rescue of CR42858 recovered the abnormal morphology

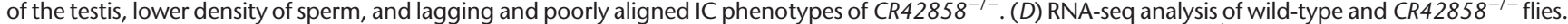
The heat map shows fold changes in expression of each of the top 18 differentially expressed genes (DEGs) in CR42858 ${ }^{-/-}$relative to those in the wild type, and the DEGs were corrected for the biological variability by combining biological replicates (NOISeqBIO method in NOISeq, version 2.14.1) (Tarazona et al. 2015). (E) Expression changes of six neighboring genes of $C R 42858^{-/-}$. RNA was isolated from testes of $C R 42858^{-/-}$and wild type and subjected to quantitative RT-PCR. RpL32 was used as an internal control. Results are represented as means \pm SEM of three biological replicates. Asterisks indicate a significant difference between the samples $(P<0.05$; $t$-test). $(F)$ Schematic illustration of in vivo functions of IncRNAs in fly spermatogenesis. LncRNAs can regulate the expression of both local and distal genes in testis, and function in post-meiotic stages of spermatogenesis.

CR42858 $8^{-/}$are caused by the deletion of regulatory DNA elements. RNA sequencing (RNA-seq) revealed that hundreds of genes were up- or down-regulated ( $\log _{2}$ Ratio| $\geq 1$ ), respectively, in $C R 42858^{-1-}$ testis in comparison with wild-type testis. These CR42858-regulated genes include protein-coding genes as well as lncRNA genes (Fig. 5D). Genes exhibiting the most dramatic ex- pression changes in the $C R 42858$ mutant testis were distant from the CR42858 locus, whereas genes located close to CR42858, including Teh1, exhibited moderate but significant down-regulation (Fig. 5E). These results further support the idea that these testisspecific lncRNAs primarily function in trans in late spermatogenesis (Fig. 5F). 
To gain insight into the transcriptional regulation of other lncRNAs, we performed massively parallel RNA-seq of testes from wild-type and four lncRNA KO strains (Supplemental Fig. S12). Consistent with the RNA-seq results of $C R 42858^{-/-}$, the expression of dozens of genes changed significantly in $C R 44585^{-1-}$, IncRNA:TS1 ${ }^{-/-}$, IncRNA:TS2 ${ }^{-/-}$, and CR45542 ${ }^{-/-}$(Supplemental Fig. S13). The lncRNA-regulated genes are located distantly from the lncRNAs, and many of them are associated with reproductive or metabolic processes, suggesting that IncRNAs control the expression of genes important for germ cell development in trans. Further studies will be needed to understand at the molecular level how testis-specific lncRNAs control the expression of neighboring and distantly located genes in the Drosophila testis.

LncRNAs with defective knockout phenotypes evolve much more slowly than those without phenotypic effects

We next investigated the origin and evolution of all testis-specific IncRNAs through sequence conservation and phylogenetic analysis. By comparative genomic analysis of 12 close Drosophila relatives, we first dated the evolutionary origin of all surveyed lncRNAs, with or without phenotypic effects, via their phylogenetic distribution. Although different internal nodes of the phylogenetic tree exhibited some fluctuations in the proportions of functional lncRNAs (18\%-57\%) (Fig. 6A), we observed a constant fraction of functional ones $(\sim 35 \%)$ within each evolutionary age group (Supplemental Table S2), including old ( $>40$ million years ago [Mya]) and young RNAs. A previous study found that $\sim 30 \%$ of new protein-coding genes quickly become essential (Chen et al. 2010). In addition, we investigated the sequence conservation of all surveyed lncRNAs and compared the conservation of the RNAs with that of protein-coding genes. Consistent with other studies (Necsulea et al. 2014), old lncRNAs were more conserved than younger ones (Supplemental Fig. S14). Overall, in comparison to protein-coding genes (median score, 0.986), IncRNAs evolved much more rapidly (Mann-Whitney $U$ test, $P$-value $<10^{-20}$ ). Furthermore, as expected, testis-biased coding DNA sequences (CDSs) (median score, 0.965) were under a slightly more relaxed selection constraint than CDSs overall (Mann-Whitney $U$ test, $P$-value $<10^{-16}$ ) (Haerty et al. 2007). Meanwhile, the group of lncRNAs whose knockouts had defective phenotypes (median score, 0.536) evolved more slowly than lncRNAs whose knockouts did not confer defects (median score, 0.353) (Mann-Whitney $U$ test, $P$-value $<10^{-20}$ ) (Fig. 6B). These results suggest that lncRNAs evolve more slowly once they acquire functional importance.

\section{Discussion}

Although a large number of lncRNAs have been identified in various tissues, especially in testis, their biological functions remain largely unexplored. In this study, we identified 128 testis-specific IncRNAs in Drosophila. Using a three-component CRISPR/Cas9based HR system, we deleted 105 lncRNAs and found that males exhibited reduced fertility and late spermatogenesis developmental defects in 33 of the deletion mutants. Our evolutionary analysis revealed that the functional lncRNAs tend to be under stronger selection constraints. Despite the fact that several studies report testis-enriched or testis-specific transcription of lncRNAs (Nam and Bartel 2012; Necsulea et al. 2014), this is one of the first studies to demonstrate the general relevance of lncRNAs to testis function.

\section{The three-component CRISPR/Cas9-based system facilitates generation of IncRNA knockout animal models}

Recently, genome-scale loss-of-function studies have indicated that IncRNAs are key regulators of cellular processes and development (Batista and Chang 2013; Mercer and Mattick 2013; Flynn and Chang 2014). Those studies were generally conducted in cell-based in vitro systems; however, phenotypes at the cellular and organismal levels are frequently discrepant. For example, loss-of-function studies of Malat1 or Dlx6os1 in mouse revealed subtle or undetectable phenotypes (Bond et al. 2009; Eißmann et al. 2012), despite the fact that these IncRNAs appear to be important at the cellular level. Therefore, the gold standard in the field is the targeted in vivo silencing or deletion of specific lncRNAs (Mattick 2013).

Compared to other strategies for generating gene deletions, our optimized CRISPR system offers several advantages. First, direct injection of the three components (Cas9 mRNA, gRNA, and HR plasmid) into Drosophila embryos greatly simplifies targeted
A

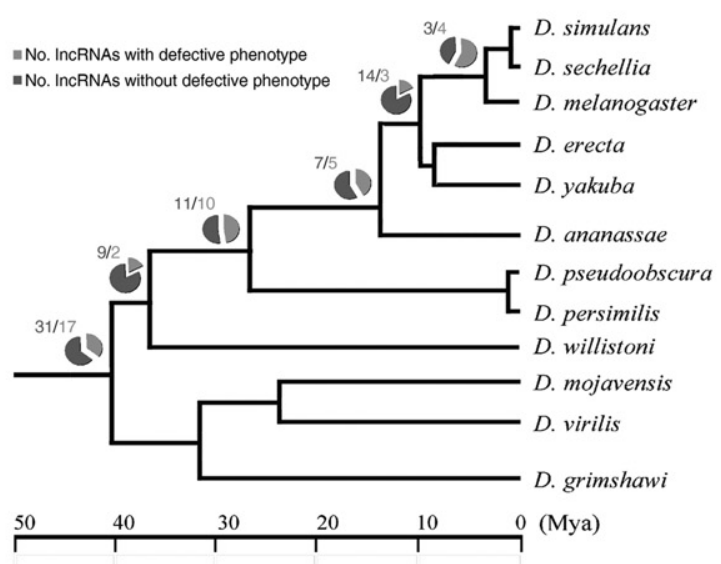

B

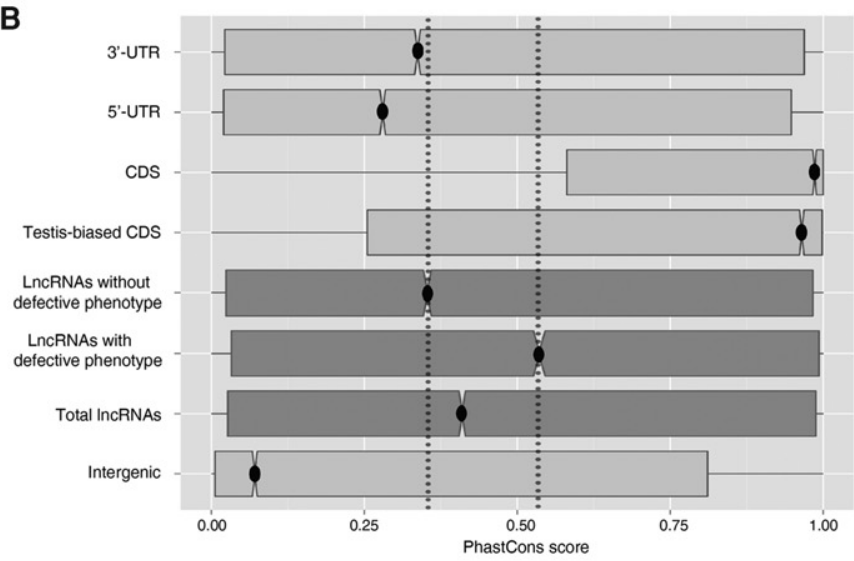

Figure 6. Evolutionary age and sequence conservation of testis-specific IncRNAs. (A) A simplified phylogenetic tree to illustrate the distribution of functional and dysfunctional IncRNAs. (B) Sequence conservation (15-way PhastCons score) for IncRNAs, with or without knockout phenotypes, and different characteristic genomic regions. PhastCons scores represent probabilities of negative selection (range between 0 and 1 ) at single-nucleotide resolution. The smaller the divergence of a DNA segment across species, the more likely it is that the segment belongs to a conserved element maintained by negative selection.

\section{Genome Research}

www.genome.org 
gene replacement. Second, our knockout method can efficiently delete genes of up to $92 \mathrm{~kb}$, and such deletions lead to a complete loss of gene function (Supplemental Fig. S3D). Third, our knockout method has a low rate of off-target effects, as demonstrated by direct sequencing of potential off-targeting loci in 22 lncRNA mutants (Supplemental Fig. S5) and rescue of six IncRNA KO mutants. Consequently, of 128 lncRNAs, we were able to successfully delete $105(82 \%)$ with an average HR frequency of $10 \%$. Taken together, these observations indicate that our CRISPR/ Cas9 system is suitable for large-scale gene deletion screens with low off-target effects in Drosophila and should also be applicable to other organisms.

The phenotype of testis-specific IncRNAs are mainly manifested in late Drosophila spermatogenesis

Recent studies show that lncRNAs are often predominantly transcribed in testis in both vertebrates (Necsulea et al. 2014) and Drosophila (Young et al. 2012), suggesting that these RNAs play similar functional roles in spermatogenesis across a broad range of animal taxa. However, it remains unclear whether lncRNAs are truly involved in male reproduction in vivo. Three mouse lncRNAs are involved in spermatogenesis in vitro (Zhang et al. 2010; Ni et al. 2011; Arun et al. 2012), but the aforementioned Pldi RNA represents the only case in which functional significance in spermatogenesis has been demonstrated in IncRNA knockout models (Heinen et al. 2009). Thus, we attempted to survey the functional roles of lncRNAs in spermatogenesis using our optimized CRISPR system.

We found that 33 lncRNA knockouts exhibited developmental defects in late spermatogenesis, resulting in low or no male fertility. The mutant phenotypes in late germ cell development indicate that these testis-specific lncRNAs play critical roles in the regulation of nuclear condensation and sperm individualization. During late spermatogenesis in both mammals and Drosophila, spermatids need to remodel and condense chromatin by replacing histones with protamines and also require the removal of excess cytoplasm for individualization (Rathke et al. 2010). CR44455/6, CR45542, and CR44420 mutant testes exhibited scattered nuclei and round uncondensed nuclei, whereas IncRNA:TS1 and CR43484 mutant testes exhibited a crumpled nucleus phenotype similar to those of protamine mutants (Rathke et al. 2010). In regard to sperm individualization, the testes of 19 lncRNA KO mutants, including $C R 42858^{-/-}$and $C R 43282^{-/-}$, exhibited defects in coordinated actin cone movement, resulting in poorly aligned or lagging ICs. Similar phenotypes have been reported for the mutants in the genes encoding the testis-specific proteasome subunit Pros $\alpha 6 \mathrm{~T}$, myosin VI, myosin V, and dynein (Hicks et al. 1999; Li et al. 2004; Mermall et al. 2005; Zhong and Belote 2007). It remains to be determined whether these lncRNAs are directly functional in late spermatogenesis or instead play a role in the early spermatogenesis that is only manifest in the late stage.

Like protein-coding genes, IncRNAs also exhibit redundancy of function. For example, the male-specific lncRNAs roX 1 and $r o X 2$ paint the $\mathrm{X}$ Chromosome of male fly, thereby contributing to equalization of X Chromosome-linked gene expression. Flies lacking roX1 or roX2 separately have no phenotype, whereas simultaneous removal of both roX 1 and roX2 causes a striking malespecific reduction in viability, indicating that these lncRNAs are functionally redundant (Meller and Rattner 2002). Similarly, some IncRNAs without phenotypes in this study may have redundant counterparts elsewhere in the genome. Meanwhile, the
lncRNAs without discernable phenotypes in this study should be further investigated using more sensitive assays, such as sperm exhaustion techniques and sperm competence tests (Yeh et al. 2012).

\section{Testis-specific IncRNAs affect late spermatogenesis primarily by regulating gene expression in trans}

The next obvious question is how lncRNAs affect late spermatogenesis. Our RNA-seq results revealed that lncRNA CR42858 controls the expression of hundreds of genes, most of which are highly expressed in the testis or are testis-specific. These differentially transcribed genes consisted of both coding genes and IncRNAs, suggesting that the general role of testis-specific lncRNAs in late spermatogenesis may involve transcriptional regulation, as proposed for other functionally characterized lncRNAs, e.g., Paupar and Pantr1 (Vance et al. 2014; Goff et al. 2015).

Some lncRNAs regulate the transcription of neighboring genes in a cis-acting manner (Lai et al. 2013; Melo et al. 2013), whereas others regulate gene expression in trans, e.g., the TP53-induced lncRNAs Dlx6os1 and Hotair (Feng et al. 2006; Huarte et al. 2010; Chu et al. 2011). We showed that CR42858 could regulate the expression of neighboring genes as well as many more distant genes. This alteration of transcription of both neighboring and distal genes upon deletion of an lncRNA is consistent with a recent study of mouse lncRNAs (Goff et al. 2015). To determine whether these testis-specific lncRNAs primarily function in cis or in trans, we performed rescue experiments on six lncRNA deletion mutants by inserting the rescue transgenes either in the endogenous locations (in cis) or in other genomic locations (in trans). Strikingly, the transgenes could rescue the spermatogenesis defects in the six lncRNA mutants both in cis and in trans, suggesting that these testis-specific lncRNAs affect late spermatogenesis by regulating the expression of target genes in trans.

\section{Separating RNA-dependent IncRNA functions from DNA sequence-dependent effects}

Three independent experiments were performed to discriminate between RNA and DNA sequence-dependent effects. First, in situ rescue experiments demonstrated that the defective phenotypes of $C R 42858^{-/-}$were due to loss of RNA-dependent lncRNA functions rather than to loss of DNA regulatory elements on the CR42858 promoter or CR42858 DNA sequences (Supplemental Fig. S11). Second, the results of RNAi against nine lncRNAs with clear knockout phenotypes revealed that the CR44585, CR43416, and CR44456 knockdown phenotypes were similar to the phenotypes of the corresponding knockouts (Supplemental Fig. S15), indicating that the phenotypes of some lncRNA KOs were indeed due to the removal of the lncRNA transcripts rather than the absence of the endogenous DNA. Third, transgenic rescue experiments on six lncRNAs showed that the spermatogenesis defects in the knockouts could be rescued by expression of the IncRNAs in trans (Fig. 5A; Supplemental Fig. S10). Collectively, this evidence argues that the phenotypes of these IncRNA KO mutants are more likely to be due to the loss of the lncRNA transcripts themselves than to changes in chromosomal DNA sequences. However, we cannot entirely rule out the possibility that DNA regulatory elements play a role in all lncRNA mutants. Further investigation will be required to more rigorously distinguish RNA from DNA sequence-dependent effects. 
Functionality may be only accumulated within a constant proportion of IncRNAs

Although dozens of lncRNAs have been implicated in various biological processes (Pauli et al. 2011; Batista and Chang 2013; Carpenter et al. 2013), the functions of the vast majority of other putative lncRNAs are largely unexplored, and it remains unclear how many lncRNAs are functional (Moran et al. 2012; Doolittle 2013). One conservative but reliable benchmark for the functionality of biological macromolecules is their conservation over the course of evolution (Graur et al. 2013). Indeed, our results showed that in testis, IncRNAs with a defective KO phenotype were more conserved than those without such a phenotype (Fig. 6B). The conservation levels for protein-coding genes and intergenic regions were consistent with expectations defined in other studies (Necsulea et al. 2014).

Remarkably, among testis-specific lncRNAs, the proportion of functional lncRNAs was similar along the sampled evolutionary ages (Supplemental Table S2), suggesting that, as in the formation of essential genes (Chen et al. 2010), a constant proportion of young lncRNAs quickly acquire important functions. Furthermore, although our analysis was limited to testis-biased lncRNAs, we hypothesize that across the entire pool of lncRNAs, functional sequences may be likely to accumulate in a stationary tempo and maintained at a constant proportion, around 30\%. More comprehensive studies combining functional and evolutionary analysis will provide further insights into this issue.

In summary, we developed an efficient CRISPR/Cas9-based gene deletion system to systematically delete 105 testis-lncRNAs in Drosophila, of which 31\% exhibited strong phenotypes, especially in late spermatogenesis, and an equivalent proportion quickly becoming functional independent of their age. Thus, our study provides important insights into the functions and evolution of tissue-specific lncRNAs, and the mutant IncRNAs generated by this study will be a valuable resource for future studies of spermatogenesis and the functions of lncRNA.

\section{Methods}

\section{Curation of known and novel IncRNAs in fly}

We first collected the annotated ncRNAs from FlyBase r5.45 and then adopted a machine learning method to predict novel IncRNAs in Drosophila (Lu et al. 2011; Gerstein et al. 2014; Hu et al. 2015). In this method, we used multiple features (e.g., sequence, structure, and expression data) to train a random forest model. The model used the lncRNAs annotated in FlyBase as the training set, and then made predictions throughout the whole genome (Hu et al. 2015). Subsequently, the annotated (known) and predicted novel lncRNAs were filtered and classified based on their genomic locations. They were subtyped into antisense, intronic, ambiguous, and intergenic ncRNAs (Di et al. 2014). To remove the ambiguity, we only retained intergenic lncRNAs (i.e., lincRNAs) for further studies. To select testis-specific lncRNAs, we used expression profiles derived from ENCODE RNA-seq data (for details, see Hu et al. 2015, supplemental table). These testis-specific IncRNAs were verified by qRT-PCR and whole-mount RNA in situ hybridization as described in the Supplemental Methods.

\section{Generation of IncRNA knockout flies}

In vitro transcription of Cas 9 mRNA was performed using the Sp6 mMESSAGE mMACHINE Kit (Ambion), according to Yu et al. (2013). In vitro transcription of the designed gRNAs was per- formed using the RiboMAX Large Scale RNA Production SystemsT7 Kit (Promega). Purified Cas 9 mRNA, gRNA, and donor plasmid were mixed at final concentrations of $1 \mu \mathrm{g} / \mu \mathrm{L}, 50 \mathrm{ng} / \mu \mathrm{L}$, and 0.8 $\mu \mathrm{g} / \mu \mathrm{L}$, respectively, followed by injection into w1118 embryos (Supplemental Table S3). The details of donor plasmid construction and gRNA design, Cas9/gRNA-mediated lncRNA deletion screen, in cis and in trans rescue of lncRNA knockout flies, and off-target analysis are listed in the Supplemental Methods.

\section{Qualitative fertility assays}

Fertility tests for males were always performed in batches of 15 . For each lncRNA knockout mutant, one lncRNA homozygous mutant virgin male was placed in a vial with one wild-type virgin female at $27.5^{\circ} \mathrm{C}$. For the next $15 \mathrm{~d}$, the flies from each mating were transferred to new vials every $24 \mathrm{~h}$. Upon eclosion, all progeny from each vial were counted (Sitnik et al. 2014). The average number of flies per parental pair and standard errors were calculated for each combination of genotypes. The details of testis imaging, phalloidin staining, and immunohistochemistry are listed in the Supplemental Methods.

\section{RNA-seq analysis}

RNA libraries were prepared for sequencing using standard Illumina protocols. Library products were sequenced on an Illumina HiSeq 2000 at the BGI (http://www.genomics.cn/index). The differentially expressed genes between two samples with biological replicates were identified using NOISeq, version 2.14.1 (Tarazona et al. 2015). See the Supplemental Methods for more details.

\section{Evolutionary age and sequence conservation analysis of IncRNAs}

For IncRNAs with successful KO mutants, we estimated their evolutionary age based on their phylogenetic distribution on a reference tree: $((((($ droMel, $($ droSim,droSec $)), \quad($ droYak, droEre $))$, droAna), (droPer, droPse)), droWil), ((droVir, droMoj), droGri)) (Stark et al. 2007). All lncRNAs emerging more than 40 million years ago were regarded as old lncRNAs (Chen et al. 2010), and the young IncRNAs were further divided into two or three age groups to calculate the relative proportion of functional lncRNAs and study the emergence of IncRNA functionalization. Sequence conservation was assessed by PhastCons score. See the Supplemental Methods for additional details.

\section{Data access}

The RNA-seq data sets generated in this study have been submitted to the NCBI Sequence Read Archive (SRA; http://www.ncbi.nlm. nih.gov/sra/) under accession numbers SRX1542553 for IncRNA:TS1 knockout, SRX1542555 for IncRNA:TS2 knockout, SRX1512980 for CR44585 knockout, SRX1542556 for CR45542 knockout, SRX1542557 for CR42858 knockout, and SRX1542554 for wild type.

\section{Acknowledgments}

This work was supported by grants from the National Natural Science Foundation of China (31171278, 31271542). We thank Dr. Ting Xie, Dr. Xiaohua Shen, and Dr. Yong Zhang for critical suggestions on manuscript preparation.

Author contributions: K.W. and G.G. conceived the project, designed the experiments, and wrote the manuscript; L.Y., K.W., M. W., Z.X., and X.Z. generated lncRNA mutants using the CRISPR system; L.L., W.Z., J.Z., X.B., and J.D. constructed the plasmids;

\section{Genome Research}

www.genome.org 
T.X. and Q.Z. performed evolutionary analyses; C.D. and Z.J.L. predicted novel lncRNAs in Drosophila; K.W., L.Y., D.M., and L.L. performed phenotype classifications and analyzed the data.

\section{References}

Arun G, Akhade VS, Donakonda S, Rao MR. 2012. mrhl RNA, a long noncoding RNA, negatively regulates Wnt signaling through its protein partner Ddx5/p68 in mouse spermatogonial cells. Mol Cell Biol 32: 3140-3152.

Batista PJ, Chang HY. 2013. Long noncoding RNAs: cellular address codes in development and disease. Cell 152: 1298-1307.

Bond AM, VanGompel MJ, Sametsky EA, Clark MF, Savage JC, Disterhoft JF, Kohtz JD. 2009. Balanced gene regulation by an embryonic brain ncRNA is critical for adult hippocampal GABA circuitry. Nat Neurosci 12: 1020-1027.

Brown JB, Boley N, Eisman R, May GE, Stoiber MH, Duff MO, Booth BW, Wen J, Park S, Suzuki AM. 2014. Diversity and dynamics of the Drosophila transcriptome. Nature 512: 393-399.

Carpenter S, Aiello D, Atianand MK, Ricci EP, Gandhi P, Hall LL, Byron M, Monks B, Henry-Bezy M, Lawrence JB. 2013. A long noncoding RNA mediates both activation and repression of immune response genes. Science 341: 789-792.

Chen S, Zhang YE, Long M. 2010. New genes in Drosophila quickly become essential. Science 330: 1682-1685.

Chu C, Qu K, Zhong FL, Artandi SE, Chang HY. 2011. Genomic maps of long noncoding RNA occupancy reveal principles of RNA-chromatin interactions. Mol Cell 44: 667-678.

Cong L, Ran FA, Cox D, Lin S, Barretto R, Habib N, Hsu PD, Wu X, Jiang W, Marraffini LA. 2013. Multiplex genome engineering using CRISPR/Cas systems. Science 339: 819-823.

Di C, Yuan J, Wu Y, Li J, Lin H, Hu L, Zhang T, Qi Y, Gerstein MB, Guo Y. 2014. Characterization of stress-responsive lncRNAs in Arabidopsis thaliana by integrating expression, epigenetic and structural features. Plant J 80: $848-861$.

Djebali S, Davis CA, Merkel A, Dobin A, Lassmann T, Mortazavi A, Tanzer A Lagarde J, Lin W, Schlesinger F. 2012. Landscape of transcription in human cells. Nature 489: 101-108.

Doolittle WF. 2013. Is junk DNA bunk? A critique of ENCODE. Proc Natl Acad Sci 110: 5294-5300.

Eißmann M, Gutschner T, Hämmerle M, Günther S, Caudron-Herger M Groß M, Schirmacher P, Rippe K, Braun T, Diederichs S. 2012. Loss of the abundant nuclear non-coding RNA MALAT1 is compatible with life and development. RNA Biol 9: 1076-1087.

Feng J, Bi C, Clark BS, Mady R, Shah P, Kohtz JD. 2006. The Evf-2 noncoding RNA is transcribed from the Dlx-5/6 ultraconserved region and functions as a Dlx-2 transcriptional coactivator. Genes Dev 20: 1470-1484.

Flynn RA, Chang HY. 2014. Long noncoding RNAs in cell-fate programming and reprogramming. Cell Stem Cell 14: 752-761.

Garneau JE, Dupuis MÈ, Villion M, Romero DA, Barrangou R, Boyaval P, Fremaux C, Horvath P, Magadán AH, Moineau S. 2010. The CRISPR/ Cas bacterial immune system cleaves bacteriophage and plasmid DNA. Nature 468: 67-71.

Gerstein MB, Rozowsky J, Yan KK, Wang D, Cheng C, Brown JB, Davis CA, Hillier L, Sisu C, Li JJ. 2014. Comparative analysis of the transcriptome across distant species. Nature 512: 445-448.

Goff LA, Groff AF, Sauvageau M, Trayes-Gibson Z, Sanchez-Gomez DB, Morse M, Martin RD, Elcavage LE, Liapis SC, Gonzalez-Celeiro M, et al. 2015. Spatiotemporal expression and transcriptional perturbations by long noncoding RNAs in the mouse brain. Proc Natl Acad Sci 112: 6855-6862.

Gratz SJ, Ukken FP, Rubinstein CD, Thiede G, Donohue LK, Cummings AM, O'Connor-Giles KM. 2014. Highly specific and efficient CRISPR/Cas9catalyzed homology-directed repair in Drosophila. Genetics 196: 961-971.

Graur D, Zheng Y, Price N, Azevedo RB, Zufall RA, Elhaik E. 2013. On the immortality of television sets: "function" in the human genome according to the evolution-free gospel of ENCODE. Genome Biol Evol 5: 578-590.

Guttman M, Amit I, Garber M, French C, Lin MF, Feldser D, Huarte M, Zuk O, Carey BW, Cassady JP, et al. 2009. Chromatin signature reveals over a thousand highly conserved large non-coding RNAs in mammals. Nature 458: 223-227.

Haerty W, Jagadeeshan S, Kulathinal RJ, Wong A, Ravi Ram K, Sirot LK, Levesque L, Artieri CG, Wolfner MF, Civetta A, et al. 2007. Evolution in the fast lane: rapidly evolving sex-related genes in Drosophila. Genetics 177: 1321-1335.

Heinen TJ, Staubach F, Haming D, Tautz D. 2009. Emergence of a new gene from an intergenic region. Curr Biol 19: 1527-1531.
Hicks JL, Deng WM, Rogat AD, Miller KG, Bownes M. 1999. Class VI unconventional myosin is required for spermatogenesis in Drosophila. Mol Biol Cell 10: 4341-4353.

Hu L, Di C, Kai M, Yang YC, Li Y, Qiu Y, Hu X, Yip KY, Zhang MQ, Lu ZJ. 2015. A common set of distinct features that characterize noncoding RNAs across multiple species. Nucleic Acids Res 43: 104-114.

Huarte M, Guttman M, Feldser D, Garber M, Koziol MJ, Kenzelmann-Broz D, Khalil AM, Zuk O, Amit I, Rabani M. 2010. A large intergenic noncoding RNA induced by p53 mediates global gene repression in the p53 response. Cell 142: 409-419.

Iyer MK, Niknafs YS, Malik R, Singhal U, Sahu A, Hosono Y, Barrette TR, Prensner JR, Evans JR, Zhao S, et al. 2015. The landscape of long noncoding RNAs in the human transcriptome. Nat Genet 47: 199-208.

Jinek M, Chylinski K, Fonfara I, Hauer M, Doudna JA, Charpentier E. 2012. A programmable dual-RNA-guided DNA endonuclease in adaptive bacterial immunity. Science 337: 816-821.

Lai F, Orom UA, Cesaroni M, Beringer M, Taatjes DJ, Blobel GA, Shiekhattar R. 2013. Activating RNAs associate with Mediator to enhance chromatin architecture and transcription. Nature 494: 497-501.

Lee JT. 2012. Epigenetic regulation by long noncoding RNAs. Science 338: 1435-1439.

Lee JT, Bartolomei MS. 2013. X-inactivation, imprinting, and long noncoding RNAs in health and disease. Cell 152: 1308-1323.

Lehmann R, Nüsslein-Volhard C. 1986. Abdominal segmentation, pole cell formation, and embryonic polarity require the localized activity of oskar, a maternal gene in Drosophila. Cell 47: 141-152.

Li MG, Serr M, Newman EA, Hays TS. 2004. The Drosophila tctex-1 light chain is dispensable for essential cytoplasmic dynein functions but is required during spermatid differentiation. Mol Biol Cell 15: 3005-3014.

Lu ZJ, Yip KY, Wang G, Shou C, Hillier LW, Khurana E, Agarwal A, Auerbach R, Rozowsky J, Cheng C. 2011. Prediction and characterization of noncoding RNAs in C. elegans by integrating conservation, secondary structure, and high-throughput sequencing and array data. Genome Res 21: 276-285.

Ma Z, Liu Z, Huang X. 2010. OSBP- and FAN-mediated sterol requirement for spermatogenesis in Drosophila. Development 137: 3775-3784.

Mali P, Yang L, Esvelt KM, Aach J, Guell M, DiCarlo JE, Norville JE, Church GM. 2013. RNA-guided human genome engineering via Cas9. Science 339: $823-826$.

Maruyama T, Dougan SK, Truttmann MC, Bilate AM, Ingram JR, Ploegh HL. 2015. Increasing the efficiency of precise genome editing with CRISPRCas9 by inhibition of nonhomologous end joining. Nat Biotechnol 33: 538-542.

Mattick JS. 2013. Probing the phenomics of noncoding RNA. eLife 2: e01968.

Meller VH, Rattner BP. 2002. The roX genes encode redundant male-specific lethal transcripts required for targeting of the MSL complex. EMBO J 21: 1084-1091.

Melo CA, Drost J, Wijchers PJ, van de Werken H, de Wit E, Vrielink JAO, Elkon R, Melo SA, Léveillé N, Kalluri R. 2013. eRNAs are required for p53-dependent enhancer activity and gene transcription. Mol Cell 49: 524-535.

Mercer TR, Mattick JS. 2013. Structure and function of long noncoding RNAs in epigenetic regulation. Nat Struct Mol Biol 20: 300-307.

Mermall V, Bonafe N, Jones L, Sellers JR, Cooley L, Mooseker MS. 2005. Drosophila myosin $\mathrm{V}$ is required for larval development and spermatid individualization. Dev Biol 286: 238-255.

Moran VA, Perera RJ, Khalil AM. 2012. Emerging functional and mechanistic paradigms of mammalian long non-coding RNAs. Nucleic Acids Res 40: 6391-6400.

Morris KV, Mattick JS. 2014. The rise of regulatory RNA. Nat Rev Genet 15: 423-437.

Nam JW, Bartel DP. 2012. Long noncoding RNAs in C. elegans. Genome Res 22: 2529-2540.

Necsulea A, Soumillon M, Warnefors M, Liechti A, Daish T, Zeller U, Baker JC, Grützner F, Kaessmann H. 2014. The evolution of lncRNA repertoires and expression patterns in tetrapods. Nature 505: 635-640.

Ni MJ, Hu ZH, Liu Q, Liu MF, Lu MH, Zhang JS, Zhang L, Zhang YL. 2011. Identification and characterization of a novel non-coding RNA involved in sperm maturation. PLoS One 6: e26053.

Pauli A, Rinn JL, Schier AF. 2011. Non-coding RNAs as regulators of embryogenesis. Nat Rev Genet 12: 136-149.

Ponting CP, Oliver PL, Reik W. 2009. Evolution and functions of long noncoding RNAs. Cell 136: 629-641.

Rathke C, Baarends WM, Jayaramaiah-Raja S, Bartkuhn M, Renkawitz R, Renkawitz-Pohl R. 2007. Transition from a nucleosome-based to a protamine-based chromatin configuration during spermiogenesis in Drosophila. J Cell Sci 120: 1689-1700.

Rathke C, Barckmann B, Burkhard S, Jayaramaiah-Raja S, Roote J, Renkawitz-Pohl R. 2010. Distinct functions of Mst77F and protamines 


\section{Wen et al.}

in nuclear shaping and chromatin condensation during Drosophila spermiogenesis. Eur J Cell Biol 89: 326-338.

Sauvageau M, Goff LA, Lodato S, Bonev B, Groff AF, Gerhardinger C, Sanchez-Gomez DB, Hacisuleyman E, Li E, Spence M. 2013. Multiple knockout mouse models reveal lincRNAs are required for life and brain development. eLife 2: $\mathrm{e} 01749$.

Sitnik JL, Francis C, Hens K, Huybrechts R, Wolfner MF, Callaerts P. 2014. Neprilysins: an evolutionarily conserved family of metalloproteases that play important roles in reproduction in Drosophila. Genetics 196: 781-797.

Stark A, Lin MF, Kheradpour P, Pedersen JS, Parts L, Carlson JW, Crosby MA, Rasmussen MD, Roy S, Deoras AN, et al. 2007. Discovery of functional elements in 12 Drosophila genomes using evolutionary signatures. Nature 450: $219-232$

Tarazona S, Furió-Tarí P, Turrà D, Pietro AD, Nueda MJ, Ferrer A, Conesa A. 2015. Data quality aware analysis of differential expression in RNA-seq with NOISeq R/Bioc package. Nucleic Acids Res 43: e140.

Texada MJ, Simonette RA, Johnson CB, Deery WJ, Beckingham KM. 2008. Yuri gagarin is required for actin, tubulin and basal body functions in Drosophila spermatogenesis. J Cell Sci 121: 1926-1936.

Ulitsky I, Bartel DP. 2013. lincRNAs: genomics, evolution, and mechanisms. Cell 154: 26-46.

Vance KW, Sansom SN, Lee S, Chalei V, Kong L, Cooper SE, Oliver PL, Ponting CP. 2014. The long non-coding RNA Paupar regulates the expression of both local and distal genes. EMBO J 33: 296-311.

Wiedenheft B, Sternberg SH, Doudna JA. 2012. RNA-guided genetic silencing systems in bacteria and archaea. Nature 482: 331-338.
Yeh SD, Do T, Chan C, Cordova A, Carranza F, Yamamoto EA, Abbassi M, Gandasetiawan KA, Librado P, Damia E. 2012. Functional evidence that a recently evolved Drosophila sperm-specific gene boosts sperm competition. Proc Natl Acad Sci 109: 2043-2048.

Yin Y, Yan P, Lu J, Song G, Zhu Y, Li Z, Zhao Y, Shen B, Huang X, Zhu H. 2015. Opposing roles for the lncRNA Haunt and its genomic locus in regulating HOXA gene activation during embryonic stem cell differentiation. Cell Stem Cell 16: 504-516.

Young RS, Marques AC, Tibbit C, Haerty W, Bassett AR, Liu JL, Ponting CP. 2012. Identification and properties of 1,119 candidate lincRNA loci in the Drosophila melanogaster genome. Genome Biol Evol 4: 427-442.

Yu Z, Ren M, Wang Z, Zhang B, Rong YS, Jiao R, Gao G. 2013. Highly efficient genome modifications mediated by CRISPR/Cas9 in Drosophila. Genetics 195: 289-291.

Yu C, Liu Y, Ma T, Liu K, Xu S, Zhang Y, Liu H, La Russa M, Xie M, Ding S. 2015. Small molecules enhance CRISPR genome editing in pluripotent stem cells. Cell Stem Cell 16: 142-147.

Zhang L, Lu H, Xin D, Cheng H, Zhou R. 2010. A novel ncRNA gene from mouse chromosome 5 trans-splices with Dmrt1 on chromosome 19. Biochem Biophys Res Commun 400: 696-700.

Zhong L, Belote JM. 2007. The testis-specific proteasome subunit Pros $\alpha 6 \mathrm{~T}$ of D. melanogaster is required for individualization and nuclear maturation during spermatogenesis. Development 134: 3517-3525.

Received September 14, 2015; accepted in revised form July 12, 2016.

\section{Genome Research}

www.genome.org 


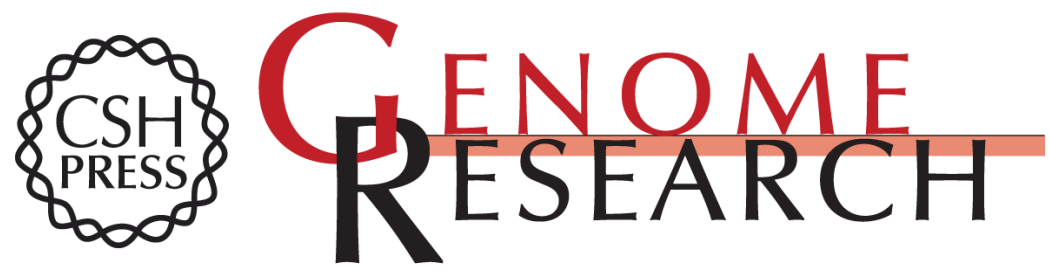

\section{Critical roles of long noncoding RNAs in Drosophila spermatogenesis}

Kejia Wen, Lijuan Yang, Tuanlin Xiong, et al.

Genome Res. 2016 26: 1233-1244 originally published online August 11, 2016 Access the most recent version at doi:10.1101/gr.199547.115

\section{Supplemental} Material

References

Creative Commons

License

Email Alerting Service
http://genome.cshlp.org/content/suppl/2016/08/11/gr.199547.115.DC1

This article cites 65 articles, 25 of which can be accessed free at: http://genome.cshlp.org/content/26/9/1233.full.html\#ref-list-1

This article is distributed exclusively by Cold Spring Harbor Laboratory Press for the first six months after the full-issue publication date (see

http://genome.cshlp.org/site/misc/terms.xhtml). After six months, it is available under a Creative Commons License (Attribution-NonCommercial 4.0 International), as described at http://creativecommons.org/licenses/by-nc/4.0/.

Receive free email alerts when new articles cite this article - sign up in the box at the top right corner of the article or click here.

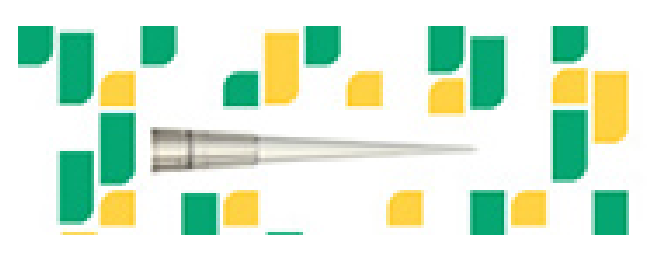

Focused on your science.

Jコగ

SCIENTIFIC

saos or seisnes

To subscribe to Genome Research go to: https://genome.cshlp.org/subscriptions 